\title{
Crystallization of metals in the ultrasonic field
}

\author{
Pavel Danilov ${ }^{1, *}$, Olga Kudryashova ${ }^{2}$, and Marina Khmeleva ${ }^{1}$ \\ ${ }^{1}$ Tomsk State University, 634050, Tomsk, Lenin avenue, 36, Russian Federation \\ ${ }^{2}$ Tomsk State University, 634050, Tomsk, Lenin avenue, 36, Russian Federation; Institute for Problems of Chemical and \\ Energetic Technologies of the Siberian Branch of the Russian Academy of Sciences, 659322, Altai krai, Biysk, \\ Socialisticheskaya str., 1, Russian Federation
}

\begin{abstract}
In the manuscript process of crystallization of metals at ultrasonic influence is considered. The known models of crystallization under the influence of ultrasound are investigated. By means of calculations influence of parameters of ultrasonic influence and characteristics of crystals of metal on the received structure of alloy is established. The corresponding mathematical model of ultrasonic crushing of particles and their agglomerates in metal fusion is offered. It is determined the threshold mode of ultrasonic influence on fusion at which almost monodisperse distribution of particles by the sizes is reached.
\end{abstract}

\section{Introduction}

It is known that ultrasound positively influences on the process of a crystallization of metals (the size of grain and the second phases decreases, the plasticity of metal increases). Ultrasonic processing of melts during a crystallization also promotes increase in wettability and a deaglomeration of particles [1] (due to formation on surfaces of capillary cracks of nonmetallic solids of cavitational bubbles which blow up, releasing the energy generating impulses of high pressure), is one of the most efficient ways of decontamination of alloy [2].

A lot of manuscripts are devoted to studying of structure and properties of melts of the metals processed by ultrasound [1-9]. Discovery was made [10] that under some conditions of ultrasonic processing of melts it is possible to reach extremely crushed (not dendritic) structure of casting metal.

The following facts of ultrasonic impact on a melt are experimentally found.

About the speed of a crystallization and influence of characteristics of ultrasound on it:

- process of a crystallization accelerates in all volume of a melt (sometimes on 3 orders);

- the threshold nature of acceleration with intensity of ultrasound: at increase in intensity at first crystallization process sharply accelerates, and further increase in intensity changes nothing.

About metal structural change:

- the average size of grain decreases,

- the bar structure is eliminated, peddling grain is formed,

- the nature of distribution of phases (by quantity, reduction ratio, the relative positioning) changes,

- the uniformity of an ingot increases,
- nonmetallic particles are distributed in volume basis an ingot more evenly.

On impact of ultrasound on decontamination of a melt:

- ultrasonic influence - one of the most efficient ways of decontamination (surpasses vacuum decontamination and a purge by gas);

- under the influence of the acoustic field there is a pulsation of bubbles of hydrogen, shallow bubbles unite in larger and emerge on a melt surface;

- ultrasonic decontamination allows to lower a pore space from 1.6 vol. \% to $0.3 \%$.

Despite the large volume of the obtained experimental data and existence of theoretical ideas of process of a crystallization of metals under the influence of ultrasound, the equations describing process still were not investigated. Such research would allow to reveal the optimum modes of ultrasonic influence at a crystallization of metals.

The purpose of this manuscript - to research dependences between speed of a crystallization, a threshold intensity of ultrasound (necessary for grain decrease) and physical and chemical properties of metal and characteristics of process. The research is based on the available theoretical ideas of process of a crystallization of metals under the influence of ultrasound.

\footnotetext{
Corresponding author: padanilov@gmail.com
} 


\section{The mathematical description of process of a crystallization of metal under the influence of ultrasound}

\subsection{Crystallization of metals without ultrasonic influence $[3,11]$}

According to the modern representations, a crystallization of metal comes from a melt on crystallization centers. In case of homogeneous origin of a solid phase crystallization centers arise directly in volume of a melt. However, at a melt surely there are foreign solids (insoluble impurity, casual particles, expressly injected additives). Thus, in an actual melt crystallization centers arise on such particles more often, there is a heterogeneous origin. Crystallization speed (quantity of crystallization centers in unit of time in unit volume) can be expressed by the following equation:

$$
n=C e^{-\frac{\Delta F}{R T}} e^{-\frac{U}{R T}},
$$

where $C$ - the constant of proportionality reflecting molecular and kinetic features of process, $\Delta F$ - work of formation of a nucleus of the critical size, $U$ - activation energy of process of transport, $R$ - the universal gas constant, $T$ - an absolute temperature.

Work of formation of a nucleus is defined by expression:

$$
\Delta F=\frac{A \sigma^{3}}{\Delta T^{2}}
$$

where $A$ - the parameter determined by a form of a nucleus and thermodynamic properties of substance: $A=32\left(\frac{M}{\rho}\right)^{2} \frac{T_{0}^{2}}{Q_{0}^{2}}$ (for cubic nuclei), $M, \rho-$ molecular mass and density of metal, $Q_{0}$ - heat of melting, $\sigma-$ the interfacial tension liquid-crystal, $\Delta T=T_{0}-T$ - overcooling of a melt, $T_{0}$ - melting point, $T$ - temperature of origin of a crystallization center.

The equation (1) has two activation thresholds:

- the first is bound to formation of crystallization centers; - the second - to phase change of the crystallizing metal.

At ultrasonic influence both thresholds that has to lead to increase in nucleation rate and as result - to formation of more fine grained structure of an ingot can be lowered.

Decrease of activation energy of process of transport of $U$ is possible owing to an intensification of diffusion processes that occurs at ultrasonic influence. At the same time communication of a diffusion coefficient with counting rate, as a first approximation, can be considered the linear [12].

In work [12] it is shown that at acoustic influence diffusion rate in liquid changes, first of all, due to activation energy change. Decomposing an exponential curve $e^{-\frac{U}{R T}}$ in a row and neglecting terms of the second order it is possible to show that the linear relation of diffusion rate from counting rate is reached at the linear decreasing relation of a threshold of activation from counting rate.

In problems of a crystallization of metals, the modified micro and nanoparticles especially the particularly important becomes heterogeneous origin of crystals as particles, injected into a melt, are crystallization centers. Then work of formation of a crystal is defined by expression [3]:

$$
\Delta F^{\prime}=\Delta F \frac{2-2 \cos \theta-\sin ^{2} \theta \cos \theta}{4},
$$

where $\theta$ - limiting wetting angle.

The analysis of formulas (1-3) shows that decrease of the interfacial tension liquid-crystal $\sigma$, decrease of a limiting wetting angle $\theta$ leads to decrease of work of formation of a nucleus, and it, in turn, has to lead to increase of nucleation of crystallization centers rate.

Using expressions (1-2), we will construct dependence of the relative speed of a crystallization of $n / C$ on temperature of overcooling (Figure 1).

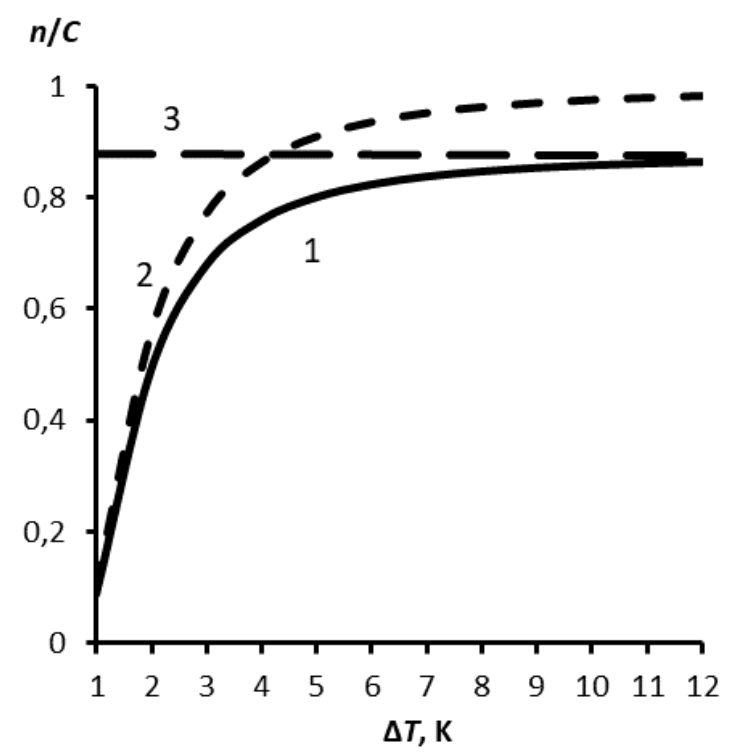

Fig. 1. Dependence of the relative speed of a crystallization $n / C$ (1) and its components $e^{-\frac{\Delta F}{R T}}(2)$ and $e^{-\frac{U}{R T}}$ (3) from overcoolin.

Also we will construct dependence of the relative speed of a crystallization of $n / C$ on ultrasound power $I$ (Figure 2). 


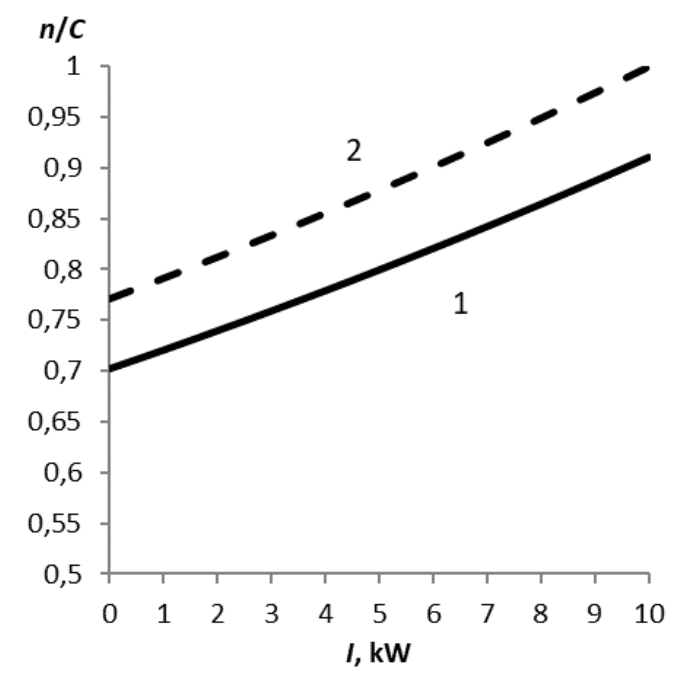

Fig. 2. Dependence of the relative speed of a crystallization of $n / C$ on the power of ultrasonic influence: 1 - without change of limiting wetting angle, $2-$ when $\theta=0$.

The Figure 2 shows the linear relation of speed of a crystallization from ultrasound power that coincides with work [12] conclusions. With counting rate body height crystallization speed significantly increases in comparison with lack of influence thanks to an intensification of processes of diffusion.

Ultrasonic influence and catalytic effects of substance of the modifying particles (at their importation in a melt) can strongly reduce the size of a limiting wetting angle. In this case, according to expression (3), work of formation of a crystallization tends to zero [9], and crystallization speed even more increases (a curve 2 in the Figure 2). However influence of this effect affects only at small temperatures of overcooling of a melt.

\subsection{Structural changes at a crystallization of metals in the presence of the ultrasonic field}

As numerous experiments, the threshold power necessary for noticeable grain refinement, sometimes below, than a cavitation threshold show. It means that for improvement of structure of metal the cavitational mode is not an indispensable condition. However, arising, the cavitation promotes grain decrease that for some metals determines threshold power.

The dispersion of crystals is influenced, apparently, by forces of a viscid sliding friction in the ultrasonic field operating in a melt [13]. Forces of a viscid sliding friction can destroy crystals and particles in two-phase system a melt - the modifying particles.

Considering the crystal which is at the front crystallizations by length of $l$ and radius $r$ in work [3] it is shown that its destruction requires pressure determined by expression:

$$
P=\frac{1}{4} \sigma_{s t} \frac{r^{2}}{l^{2}}
$$

where $\sigma_{s t}-$ ultimate strength of material at a temperature, the close to melting point.
It should be noted that durability of semisolid metals can be much lower, than in solidity. So, for example, ultimate strength of A356 alloy decreases from $157 \mathrm{MPa}$ in solidity [14] up to $4.98 \mathrm{MPa}$ at a temperature of $860 \mathrm{~K}$ and to $\sim 0.01 \mathrm{MPa}$ at $880 \mathrm{~K}[15]$.

In the analysis acting on crystal in the ultrasonic field of forces in work [3] expression for calculation of the intensity of ultrasound necessary for destruction of the growing crystals is received:

$$
I_{t}=\frac{W_{l}}{2}\left(\frac{\sigma_{s t} r}{l^{2} \omega \rho_{l}}\right)^{2}
$$

where $\omega$ - ultrasound frequency, $W_{l}=\rho_{l} c$ - wave drag of a liquid phase, $\rho_{l}-$ liquid density, $c-$ sonic speed.

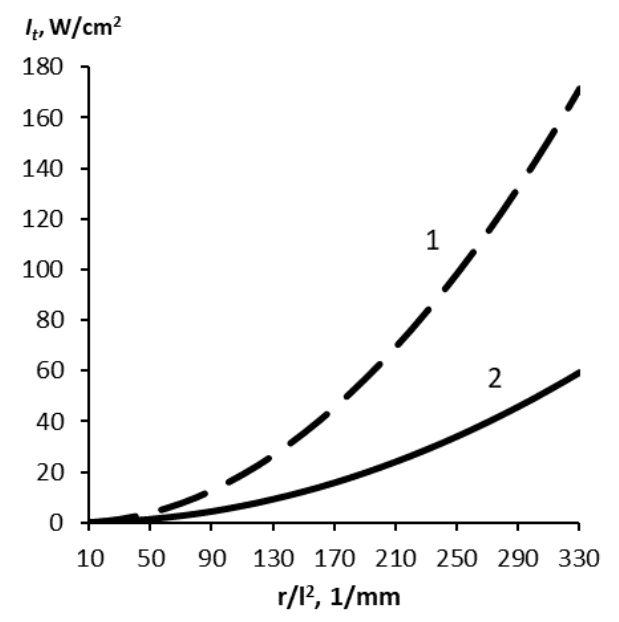

a)

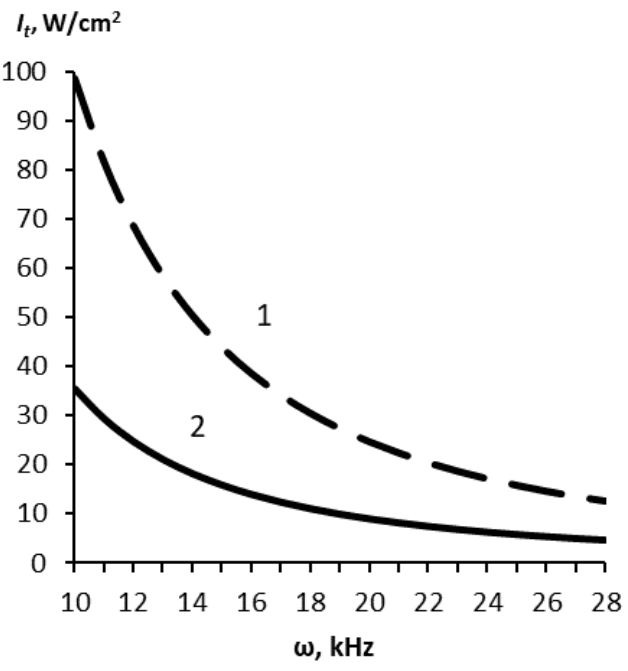

b)

Fig. 3. Dependence of a threshold intensity of destruction of crystals of metal on a dimensional factor (a): $1-\omega=10 \mathrm{kHz}$,

$2-\omega=17 \mathrm{kHz}$ and ultrasound frequency (b): $1-\frac{r}{l^{2}}$

$=2500001 / \mathrm{mm}, 2-\frac{r}{l^{2}}=1500001 / \mathrm{mm}$. 
Connection of a threshold intensity of ultrasound and wave drag of a melt (5) the linear. On the other hand, it is known that the wave drag of the environment in the mode of a developed cavitation decreases, approximately, three times [16]. Means, the cavitational mode allows to receive more fine grain in an ingot at three times lower value of counting rate with other things being equal.

The dependence of the threshold intensity of ultrasound calculated by a formula (5) on a dimensional factor $\frac{r}{l^{2}}$ and from ultrasound frequency $\omega$ is given in the Figure 3. The more extended crystal, the less is required to energy for its destruction. Increase in frequency of ultrasound also leads to essential decrease in a threshold intensity.

Thus, processing of a melt of metal ultrasound of high frequency (17 kHz and more) will allow to receive an ingot with smaller grain even at rather small ultrasound intensities. It corresponds to the experimental data obtained in works $[5,10]$.

\subsection{Dispersion of particles of powder modifiers at ultrasonic impact on a metal melt}

For input of particles of powder modifiers in a metal melt in practice use ultrasonic influence. It is supposed that the ultrasonic cavitation promotes wettability of nonmetallic particles and their best distribution in volume basis. One of hypotheses, explaining the increased efficient wettability, connects the improved access of a melt to a surface of nonmetallic particles and penetration of a melt into surface defects (capillaries) by an ultrasonic cavitation [1, 9]. The pulsing and collapsing bubbles promote a superficial cleaning of particles from adsorbed gas and impurity and also to filling of thin surface capillaries due to sound capillary effect. It promotes efficient wettability of particles and simplification of their introduction to a melt.

Besides, at introduction to a melt of metal of powder modifiers an important task is the dispersion of particles of these powders for the purpose of their uniform distribution in a melt and increases in number of crystallization centers $[17,18]$. The mathematical model of this phenomenon was not offered earlier.

Considering solids of spherical shape with $r$ radius, the distributed in molten metal and exposed to acoustic, we will receive the equation similar to the equation (5) for the extended crystals. Such equation will allow to receive values of a threshold intensity of destruction of the agglomerates and large particles injected into a melt:

$$
I_{t}=\frac{W_{l}}{2}\left(\frac{\sigma_{s t p}}{r \omega \rho_{l}}\right)^{2},
$$

where $\sigma_{s t p}-$ ultimate strength of a particle.

It must be kept in mind that ultimate strength of agglomerates of particles several orders smaller, than such size for a monolithic particle. So, adamant has high durability (an order, $500 \mathrm{MPa}$ ), but agglomerates of microparticles can collapse at $\sigma_{s t p} \sim 75 \mathrm{MPa}$ and less.

The Figure 4 shows dependence of a threshold intensity of destruction of particles of adamant in an aluminum melt under the influence of ultrasound with a frequency of $17 \mathrm{kHz}$ and $10 \mathrm{kHz}$.

Thus, shallow particles, with a radius, about 1-2 $\mu \mathrm{m}$ less, require high intensity of ultrasound for their destruction. It is worth noticing that with particle size decrease the threshold intensity sharply increases, reaching technically unrealizable values, more than 1 $\mathrm{kW} / \mathrm{cm}^{2}$. At the same time, the ultrasound frequency is lower, the intensity for destruction of particles is required above.

On the other hand, processing by ultrasound of a melt of the metal containing a suspension of microparticles at intensity below threshold promotes coagulation of these particles that is undesirable from the point of view of the practical purpose (increase in number of heterogeneous crystallization centers).

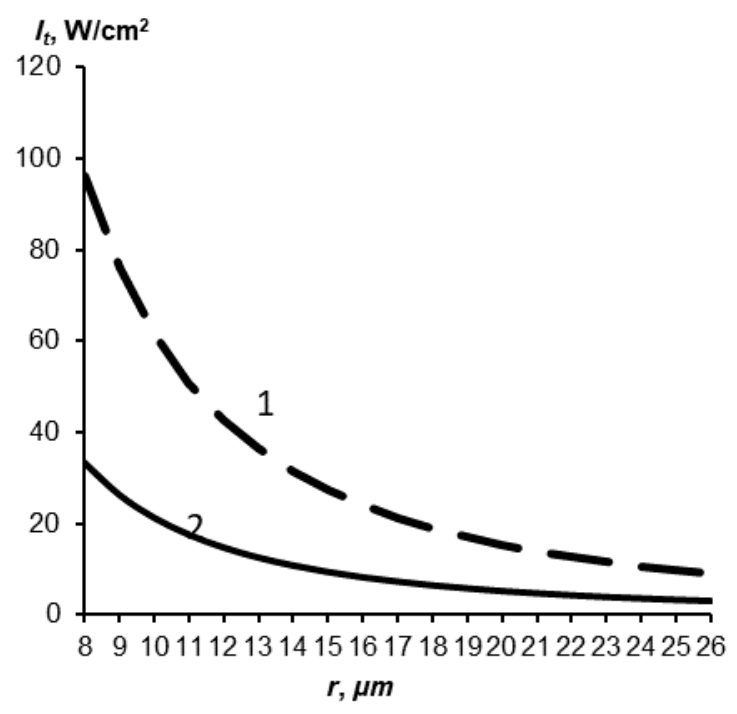

Fig. 4. Dependence of a threshold intensity of ultrasonic of destruction of particles in a metal melt from the radius of particles: $1-\omega=10 \mathrm{kHz}, 2-\omega=17 \mathrm{kHz}$.

\section{Experimental results}

Experiments on impact on a melt ultrasound at addition in a melt of modifiers show that ultrasound allows to reduce the average size of grain of alloy on an equal basis with way of addition in a melt of $\mathrm{Ti}$ and $\mathrm{Sc}$ modifiers.

Ultrasound can be applied along with addition of modifiers, increasing their effect.

Impact on an aluminum melt $(99,95$ mass $\%$ of $\mathrm{Al})$ with addition of ligatures of $\mathrm{Al}+0.18$ wt. $\% \mathrm{Zr}+$ 0.015 wt. $\%$ Ti by ultrasound with an amplitude of $40 \mu \mathrm{m}$ at a temperature of $700^{\circ} \mathrm{C}$ decrease in the average size of grain from $2500 \mu \mathrm{m}$ to $100 \mu \mathrm{m}$ [2].

In the Figure 5 the dependence of the average size of grain of alloy on the basis of aluminum from a mass fraction of maintenance of the Ti modifier is shown: 
without application of ultrasonic influence and with application.

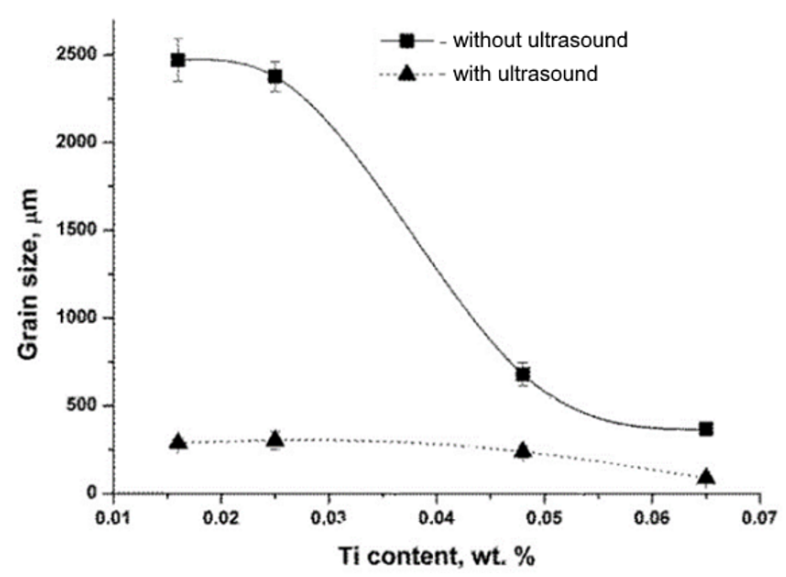

Fig. 5. Dependence of the size of grain in aluminum alloy from quantity of the added $\mathrm{Ti}$ and application of ultrasonic processing of a melt.

It is visible, the effect of ultrasonic influence is not lower than at addition of a large quantity of the modifier.

\section{Discussion}

It is possible to assume that at some parameters of ultrasound the dynamic mode at which large agglomerates will collapse to more shallow particles which then will unite in agglomerates again can be implemented. Such mode will lead to formation of monodispersible fraction of particles in a melt (possibly, with sizes about several $\mu \mathrm{m}$ ). And, for each set of parameters (frequency and counting rate, properties of particles and a melt) the reference size of such particles will be individual. Studying of such dynamic modes will give the chance of further management of properties of the received material.

On the other hand, for a dispersion of the agglomerates consisting of nanoparticles to lowdimensional will demand extremely high sizes of intensity of ultrasound. It means that receiving the metals modified by nanodimensional particles will require other mechanisms of impact on a melt.

\section{Conclusions}

1. The parametrical research of the known models of a crystallization of metal under the influence of ultrasound is conducted. Calculations revealed the threshold nature of influence of intensity of ultrasonic radiation on metal crystallization speed that corresponds to the experimental data obtained earlier.

2. Calculations according to mathematical model of subdivision of crystals of metal in the ultrasonic field are carried out. If metal is characterized by rather low interfacial tension, and crystals in the course of its low baking have more extended form, for obtaining fine grained structure of an ingot rather lower ultrasound intensities are required. Increase in frequency of ultrasound to $17 \mathrm{kHz}$ and more allow to receive fine grained structure of an ingot at significantly lower (much, in comparison with frequency $10 \mathrm{kHz}$ ) ultrasound intensities.

3. The model of ultrasonic subdivision of particles and their agglomerates in a metal melt is offered. The dispersion of agglomerates of the modifying particles requires a threshold intensity of ultrasound, inversely proportional to a square of radius of agglomerates. Processing of the melt containing powder particles in the prethreshold mode leads to coagulation of particles.

4. When processing by ultrasound of a melt of the metal modified by powder microparticles in the mode, the close to threshold it is possible to expect the dynamic equilibrium mode caused by the competition of processes of coagulation and subdivision of particles to formation of a particle size distribution, the close to monodispersible.

5. The carried-out calculations showed what for a dispersion of agglomerates of nanoparticles will demand incredibly high sizes of intensity of ultrasonic influence (about tens of $\mathrm{kW} / \mathrm{cm}^{2}$ ). It calls into question into application of ultrasonic processing for receiving nanodimensional particles in molten metals.

This work was carried out with financial support from the Ministry of Education and Science of the Russian Federation (State assignment No. 11.10533.2018/11.12).

\section{References}

1. O. Kudryashova, D. Eskin, A. Khrustalyov, S. Vorozhtsov, Russian Journal of Non-Ferrous Metals, 4 (58), 427 (2017)

2. H. Pugaa, S. Costaa, J. Barbosaa, S. Ribeirob, M. Prokic, J Mater Process Tech, 211, 1729 (2011)

3. L.D. Rozenberg, Physics and Engineering of Powerful Ultrasound. Volume 3. Physical Foundations of Ultrasonic Technology (Moscow: Scince, 1970)

4. A.P. Kapustin The influence of ultrasound on the kinetics of crystallization (Moscow: Academy of Sciences USSR, 1962).

5. G.I. Eskin, Ultrasonic Treatment of Light Alloy Melts, Second Edition (Moscow: Metallurgy, 1988).

6. B.A. Agranat, M.N. Dubrovin, N.N. Khvastovsky Fundamentals of physics and ultrasound equipment: The education guidance for higher education institutions (Moscow: Higher School, 1987)

7. S.A. Vorozhtsov, D.G. Eskin, J. Tamayo, A.B. Vorozhtsov, V.V. Promakhov, A.A. Averin, A.P. Khrustalyo, J. Metall Mater Trans, 4 (46A) 2870 (2015)

8. A.A. Kozulin, S.A. Vorozhtsov, S.S. Kulkov, S.N. Kulkov, U. Teipel, AIP Conference Proceedings 15-12 Sep 2015, Tomsk, Russia (NY : American Institute of Physics) (2015)

9. O.B. Kudryashova, A.V. Kozyrev, S.A. Vorozhtsov, Russian Physics Journal, 5 (59), 626 (2016) 
10. V.I. Dobatkin, A.F. Belov, G.I. Eskin, S.I. Borovikova, Y.G. Golder, J. Opening, Inventions, 37, 1 (1983)

11. V.I. Roldugin Physics and chemistry of a surface (Dolgoprudny: Intellect, 2011)

12. M.E. Arkhangelsky, J. UFN, 2 (92), 181 (1967)

13. I.I. Teumin, J. Problems of metallurgical science and physics of metals, 7, 375 (1962)

14. L. Arnberg, L. Bäckerud, G. Chai Dendrite Coherency, American Foundrymen's Society, Des Plaines (IL), 3, 93 (1996)

15. I.I. Novikov Hot fragility of non-ferrous metals and alloys (Moscow : Nauka, 1966)

16. G.A. Kardashev Physical methods of an intensification of processes of chemical technology (Moscow: Chemical, 1990)

17. S. Vorozhtsov, I. Zhukov, V. Promakhov, E. Naydenkin, A. Khrustalyov, A. Vorozhtsov, J. The Minerals, Metals \& Materials Society, 68, 3101 (2016) available at : https://doi.org/10.1007/s11837-016-2141-5.

18. S.A. Vorozhtsov, A.A. Kozulin, S.S. Kulkov, S.N. Kulkov, U. Teipel, J. Perspektivnye Materialy, 11, 48 (2014) 